\title{
Social cognition and work performance of persons with schizophrenia in a Chinese population
}

\author{
Panmi Lo ${ }^{\mathrm{a}}$ and Andrew M.H. Siu ${ }^{\mathrm{b}, *}$ \\ ${ }^{a}$ Castle Peak Hospital, Hospital Authority, Hong Kong, China \\ ${ }^{\mathrm{b}}$ Department of Rehabilitation Sciences, The Hong Kong Polytechnic University, Hong Kong, China
}

Received 9 July 2013

Accepted 9 October 2013

\begin{abstract}
.
BACKGROUND: Social-cognitive deficits have a significant impact on the community and vocational functioning of persons with schizophrenia.

OBJECTIVE: This study aimed to explore the relationship between social-cognitive abilities and vocational functioning in a Chinese population.

METHOD: We recruited 30 outpatients with schizophrenia to participate. We administered the Chinese Social Cognition and Screening Questionnaire (C-SCSQ) to assess Theory of Mind (ToM), attributional bias, and neurocognition; the Facial Expression Identification Test (FEIT) to assess emotion perception (EP) ability, and the Chinese Work Personality Profile (CWPP) to assess work performance in a simulated work setting.

RESULTS: ToM showed a significant negative correlation with attributional bias. The neurocognitive measure displayed a significant positive correlation with ToM and EP. The structural equation model was a good fit to the data (CFI $=0.91$, RMSEA $=0.12$, and showed that social-cognitive abilities had a significant impact $(-0.41)$ on work performance. Of the four socialcognitive domains, ToM and paranoid attributional style (PAS) contributed significantly to variations in work performance. These results support the theory that social-cognitive abilities have an impact on work performance. ToM has a positive impact whereas PAS has an adverse effect.

CONCLUSION: Persons with schizophrenia present specific deficits in their social-cognitive abilities, which have significant impact on their work performance and employability.
\end{abstract}

Keywords: Emotion perception, theory-of-mind, attributional style, vocational

\section{Introduction}

Schizophrenia is a severe psychiatric illness characterized by marked deficits in a wide array of functional abilities, including work and social functioning and independent living skills [1-3]. Work functioning is one

*Corresponding author: Andrew M. H. Siu, Department of Rehabilitation Sciences, The Hong Kong Polytechnic University, Hung Hom, Kowloon, Hong Kong, China. Tel.: +852 27666753; Fax: +852 23308656; E-mail: a.siu@ polyu.edu.hk. of the most important dimensions of recovery, and successful employment participation is linked to improvement in self-concept, self-efficacy [4], and subjective wellbeing [5], as well as symptom reduction [67]. The employability of competitive employment among persons with mental illnesses was reported to be as low as $30 \%$ [8], this figure is foreseen to be even lower among persons with schizophrenia on the basis of the more disabling nature of illness. While the value of work was high in Chinese [9], it is therefore extremely important to identify determinants of work function- 
ing that could significantly improve the employability of persons with schizophrenia in a Chinese population. Neurocognitive impairments (including attention, memory, and executive functioning) are often mentioned as good predictors of prospective work performance and future attainment of competitive employment [10-12]. In recent years, social cognition has also been proposed as an important contributor of functional and work functioning, in a way that is not redundant with neurocognition [13]. Social cognition contributed to community functioning, as a mediator between neurocognition and functioning, with effect size ranging from 0.11 to 0.28 in a systematic review of 15 studies [14]; and as a valid predictor of adjustment outcome that could not be accounted for by neurocognition alone [15-17].

Social cognition is defined as the mental operations that underlie social interactions, including perceiving, interpreting, and responding to the intentions, dispositions, and behaviors of others [18]. There is a general consensus that social cognition is closely related to neurocognition [19], but there is mounting neuroscience evidence supporting that neurocognition and social cognition are semi-independent systems for processing nonsocial and social stimuli respectively [20[21] and there is only modest association between the two constructs [19[22]. The construct of social cognition itself is broad and multifaceted, as evident from studies examining the factor structure of social cognition [23]. The current conceptualization of the social cognition construct is mainly based on the general consensus at a US National Institute of Mental Health (NIMH) workshop [24] which suggested social cognition encompasses the four key aspects of: 1) emotion perception (EP), 2) theory of mind (ToM), 3) attributional style, and 4) social perception/knowledge. A recent study further highlighted the importance of a related aspect, the tendency to jump to conclusions (JTC), which was incorporated in social cognition program [25].

First, EP refers to the ability to infer emotional information from facial expressions, vocal reflections, or a combination of both. A recent meta-analysis has highlighted that persons with schizophrenia have major deficits in EP compared with healthy controls [26]. They often display bias in recognition of others' emotions and are more likely than healthy controls to perceive negative emotions such as disgust and anger in neutral facial expressions [27]. Second, ToM refers to the ability to infer mental states and apply this understanding to predict the behavior of others [28]. Per- sons with schizophrenia perform worse in ToM tests than normal controls [29-31], with an average performance of more than one standard deviation below that of healthy controls [32]. Third, attributional style is defined as a tendency to explain the causes of life events. Some studies suggest that hostile attribution style, or the tendency to infer hostility from others' behavior, is significantly more common in patients with persecutory delusion compared with patients without this type of delusion or nonpsychiatric controls [33]. Such an attribution style is regarded as a plausible factor in the creation of paranoid thoughts [34]. Fourth, the JTC tendency refers to a predilection for making overconfident probabilistic judgments with minimal data [35]. It is often reflected in two parameters, namely how hasty the data gathering is and the tendency to make overconfident judgments, both of which can be measured.

As a whole, social-cognitive deficits and bias in persons with schizophrenia can lead to significant difficulties in social adjustment in the workplace. Deficits in EP can lead to neglect of facial cues and the misperception of coworkers' neutral emotions as anger, eliciting a hostile attitude toward others. ToM deficits may become a barrier to understanding the meaning behind instructions given by supervisors or customers. One may misunderstand what others want to communicate and fail to address their needs. The paranoid attributional style (PAS) may increase the likelihood to attribute negative events to colleagues' faults, and it together with JTC tendency, could lead to false conclusions on the colleagues' affinity, and may become a barrier to an effective working relationship. In summary, there is considerable evidence that persons with schizophrenia have significant issues with different aspects of social-cognitive abilities that possibly pose a negative impact on work performance, yet few studies have examined the specific relationship between social cognition and work functioning. Of the few available studies in this field, the results have been mixed. Social cognition could be a mediator of the relationship between neurocognition and work performance [36]. The study by Vauth et al. [37] suggested that $25 \%$ of work-related social skills were explained by social and neurocognition, but $83 \%$ of social cognition could be linked to neurocognition. Bell et al. [38] showed that social cognition and with social competence, mediated the relationship between work performance and neurocognition. In addition, there are also studies showing no significant relationship between some aspects of social cognition and global community functioning or work performance [14[38-40]. 
Several issues needed to be addressed in this field of study. Firstly, studies used a variety of definitions of social cognition, and few provided a comprehensive evaluation of social cognition. Future studies could adopt a unified definition of social cognition by the NIMH [24] and provide a comprehensive evaluation of key aspects of social cognition [23]. Secondly, many previous studies in this area used self-report measures of work and cognitive functioning that could be influenced by the insight of persons with schizophrenia [35[38]. Indicators of vocational engagement (such as wages, duration of employment) could be used to reflect work functioning, but these indicators may be influenced by external factors such as job market or level of social support. Thus, an observational-based work assessment that assesses fundamental work role requirements in simulated or naturalistic work settings would be a good choice in quantifying work performance [41]. Thirdly, there were few female subjects when compared with male subjects in many previous studies [13]. A more balanced gender mix should be provided in future studies [4243].

The present study used two social cognitive assessments to assess three out of the four consented social cognitive domains: emotion perception, theory-ofmind and attributional style [24], and another related domain, jump-to-conclusion; and used a Chinese validated work assessment, with evident predictive validity of work outcomes [44], to measure subjects' work performance in simulated work settings through direct observation. A structural equation model was constructed and tested to evaluate the overall contribution of social cognition to work performance. Further, the present study attempted to recruit subjects with a balanced proportion on the two genders. Specific research questions to answer were: 1) Are there any differences in social cognitive functioning across gender? 2) Do the four social cognitive domains correlate with each other? 3) Is there any significant relationships between the four social cognitive domains examined, EP, ToM, JTC, PAS; and work performance? 4) Are there any differences in the strength of relationships between the four social cognitive domains and work performance?

\section{Method}

This study employed a cross-sectional design that evaluates the social cognitive functioning in four social cognitive domains and the work performance in vocational rehabilitation settings among persons with schizophrenia living in the community in Hong Kong, and examines the relationship between the two constructs.

\subsection{Participants}

All the participants $(\mathrm{N}=30)$ were outpatients receiving occupational therapy services in a psychiatric hospital in Hong Kong. All participants were Cantonese speaking. The participants were included if they are aged 18-60 and have a diagnosis of schizophrenia according to the International Classification of Diseases-10 (ICD-10). The exclusion criteria included: 1) dual diagnosis such as neurological disorder, developmental disability, or substance abuse; or 2) admission to in-patient psychiatric treatment or a change in psychiatric medication within the previous 30 days.

The mean age of the participants was 41.6 years (SD $=9.23$ ), and $53 \%$ were males. The mean age of onset of schizophrenia was 24.3 years $(\mathrm{SD}=8.43)$. Most had either a secondary $(82.8 \%)$ or primary $(13.8 \%)$ education. The mean years of education was 9.0 (SD = 2.9). Two-thirds lived in their own home or with family, while the rest lived in half-way houses, supported hostels, or private hostels. The majority $(86.7 \%)$ received social security benefits. Most were participating in vocational rehabilitation at the time of the study, including hospital-based vocational training that offered job posts training, such as clerical and catering training that simulated the work tasks of future work placements, $(90.0 \%)$. The rest are receiving training in supported employment $(6.7 \%)$, and sheltered workshops $(3.3 \%)$. The mean duration of their unemployment was 47.9 months $(\mathrm{SD}=43.0)$. A small proportion of the participants $(23.3 \%)$ had had competitive or supported employment in the 2 years prior to the time of data collection. Their mean length of job tenure was 2.4 months and the mean hourly rate HK\$5.2 (US\$.67), with high variation across participants.

\subsection{Instruments}

\subsubsection{Emotion perception (EP)}

The Facial Expression Identification Test (FEIT), a procedure widely used to assess facial EP ability, was adopted in this study [45]. We used a set of 21 photos, in which 12 displayed the six basic emotions (happy, sad, angry, disgusted, fear, surprised) and 9 were neutral. All the test photos were selected from the Japanese photo bank of the Japanese and Caucasian Facial Expression of Emotion (JACFEE) and neutral faces 
(JACNeuF) photo set [46], which has been found to have acceptable levels of agreement in a Chinese sample $(\mathrm{N}=120)$ [47]. The internal consistency of the FEIT obtained from this study was 0.7 and the meanitem difficulty was 0.64 , which is similar to that obtained in previous studies [45].

A Chinese instruction sheet was prepared to enable the examiner to deliver the test instructions in a standardized manner. The 21 photos were presented to the examinee one by one, in random order, using a slide presentation on a notebook computer. Several test parameters were standardized. Firstly, the time of showing the photos and of the rest between two photos were both set at 10 seconds, with reference to the time used in previous overseas studies [45[48-50]. Secondly, the slide presentation file was presented on either a desktop computer or notebook with a screen of no less than 18 inches and at an arm's length distance between the participant and screen. This ensured that the size of the photo on the screen was similar to that found in day-today conversation. After viewing each photo, the participant was required to select one of the seven emotions to describe it. The FEIT total score is a summary of how well the participant identifies the six basic emotions and neutral emotion from the photos.

\subsubsection{Theory of mind, jump-to-conclusion, and attributional style}

The Social Cognition Screening Questionnaire (SCSQ) aims to provide evaluation of ToM, JTC, and paranoid attributional style, and was used to screen for neurocognitive deficits that would need social cognition intervention [25]. Ten second-person interpersonal vignettes, each describing an ambiguous interpersonal situation, were presented verbally to the participant. The participant was required to answer three yes/no questions and one confidence judgment question on an answer sheet for each vignette. The SCSQ has three subscales, including a neurocognitive score, a ToM score, and a JTC score. The paranoid attributional bias (PAS) score is calculated by summing the incorrectly answered perspective-taking parts in vignettes 2 , $3,5,6$, and 9 , which could reflect negative self-directed thoughts or feelings.

With the approval of the author of the original English version, we translated the SCSQ into Chinese (abbreviated as C-SCSQ) and conducted an expert panel review of its content validity. The Chinese version was further revised based on the comments and suggestions of the expert panel and some issues identified during the field test.

\subsubsection{Work performance}

We used the Chinese Work Personality Profile (CWPP) to conduct a situational assessment of the critical work role requirements of the participants. The 58item CWPP provides a profile of vocational functioning along the five subscales of task orientation, social skills, self-control, attitude, and personal appearance, and is often used to determine the appropriate vocational rehabilitation placement for the client or predict their employability [44].

\subsection{Procedures}

We obtained ethical approval to conduct the study from the Hong Kong Polytechnic University and the Hospital Authority. All potential participants were given a briefing about the research and asked to provide written informed consent. Research assistants received a three-hour training session before administering the FEIT and C-SCSQ. The principal investigator and assistants administered the tests in a quiet room in the occupational therapy department. Each session lasted for around 45 minutes. The case occupational therapists rated participants' work performance using the CWPP after observing them for at least 10 sessions in the simulated work training workshops.

\section{Results}

\subsection{Social cognitive functioning across gender}

Female subjects had significantly higher scores in FEIT total score $(t=3.35, p=0.002)$ when compared with male patient subjects, but not in control subjects. There was no significant difference in ToM, JTC and PAS across gender in either group.

\subsection{Correlations among the social-cognitive domains}

The strength of the correlations among the four social-cognitive domains ranged from 0.17 to 0.42 (Table 1). The FEIT, ToM, and neurocognitive measures were positive and the JTC and PAS negative in polarity. The correlations between FEIT and ToM, and between JTC and PAS were positive. FEIT had a negative correlation with PAS, while ToM had negative correlations with JTC and PAS. The neurocognitive measure had a significant positive correlation with FEIT ( $r=$ $0.42, p<0.05)$ and ToM $(r=0.57, p<0.01)$. 
Table 1

Correlations among the social-cognitive and neurocognitive measures

\begin{tabular}{lrrrr}
\hline Variables & FEIT & ToM & JTC & PAS \\
\hline ToM & 0.23 & & & \\
JTC & 0.25 & $-0.54^{* *}$ & & \\
PAS & -0.28 & $-0.49^{* *}$ & 0.29 & \\
Neurocognitive & $0.42^{*}$ & $0.57^{* *}$ & -0.17 & -0.33 \\
\hline
\end{tabular}

Note. Spearman's $\rho$ was estimated. FEIT $=$ Facial Expression Identification Test, ToM $=$ Theory of Mind, JTC $=$ Jump To Conclusion bias, PAS $=$ Paranoid Attributional Style. ${ }^{*} p<0.05$. ${ }^{* *} p<0.01$.

\subsection{Relationship between social-cognitive domains and work performance}

Table 2 presents the correlations between the socialcognitive, neurocognitive, and work performance measures. EP (FEIT scores) had a significant positive correlation with current work performance (CWPP subscales), in which a high correlation was found with task orientation $(r=0.60, p<0.001)$, social skills $(r=0.58, p<0.01)$, and personal presentation $(r=$ $0.51, p<0.01)$. Neurocognitive scores had a moderate positive correlation with four out of five work performance measures, with a smaller strength of $\mathrm{r}$ compared with FEIT (r ranging from 0.37 to 0.46 ). We also conducted partial correlations of pairs of relationships with age as a covariate, and most of the significant correlations remained significant. Participants who had an employment record (open or supported) within the past two years had a significantly higher score in ToM (Mann-Whitney $\mathrm{U}=29.5, \mathrm{Z}=2.576, p=0.01$ ) and lower score in JTC (Mann-Whitney $\mathrm{U}=37.5, \mathrm{Z}=$ $2.143, p=0.033$ ), when compared with the unemployed group.

We then constructed a structural equation model to examine the relationship between social-cognitive abilities and work functioning (Fig. 1). The indicators of social-cognitive abilities used included the five scores of EP (FEIT) and the ToM, PAS, JTC, and neurocognitive scores of the SCSQ. The latent variable of social-cognitive abilities is hypothesized to affect the five indicators of work performance measured by the CWPP. Initial tests showed that the model fit is high $(\mathrm{CFI}=0.85$, RMSEA $=0.15)$, which indicates a fairly good model fit. Based on the modification indexes, post hoc fitting was conducted. We added covariance among three pairs of error terms between e1 and e4, e 4 and e16, and e 8 and e10. With the addition of these paths, model fit was increased to a satisfactory level $(\mathrm{CFI}=0.91$; RMSEA $=0.12)$. The path coefficients of the structural model were significant and varied from 0.28 to 0.96 (absolute values). Social cog- nition had a significant impact on work performance $|0.41|$. The size of the covariances newly added to the three pairs of error terms were $0.26,0.35$, and 0.47 .

\section{Discussion}

This study comprehensively evaluates the four aspects of social-cognitive abilities in persons with schizophrenia in a Chinese population. The correlations among aspects of social cognition largely replicated the results of previous studies. The FEIT had a positive (but insignificant) correlation with the ToM subscale in the C-SCSQ. The strength of this correlation was within the range observed in similar studies (0.14-0.27) [36 38].

Negative correlations were observed between the positive scales of ToM and FEIT and the negative scales of the PAS and JTC. In particular, ToM showed a significant negative correlation with the PAS and JTC, which is consistent with the proposition that persons with paranoia have impaired understanding of others' intentions. This probably results in a hostile attributional style, which could not be confirmed in previous studies [34].

It is noteworthy that neurocognition had significant positive relationships with both FEIT and ToM. This implies that higher neurocognitive abilities (in this case, memory) are associated with higher ToM and the ability to perceive emotions accurately. This replicates the findings of previous studies on the positive relationship between neurocognition and ToM measures [51], and between social cognition and ToM [39 52].

The results support the proposition that socialcognitive abilities have a significant impact on work performance. Patients who had worked at some time in the previous two years had higher ToM and lower JTC scores compared with the unemployed group. The model estimated that social-cognitive abilities had a significant impact (0.41) on work performance, which is much larger than the estimate of 0.26 between social cue detection and work functioning in a recent study [36].

The estimated path coefficient between socialcognitive abilities and work performance was negative in the model, as well as the paths from these abilities to ToM, EP and neurocognitive measures. This implies that ToM, EP and neurocognitive measures contribute positively and that JTC and PAS contributed negatively to work performance. In particular, it is noted that the path coefficients linking ToM and PAS 
Table 2

Correlations Between Social Cognitive, Neurocognitive, and Work Performance Measures $(\mathrm{N}=30)$

\begin{tabular}{lccccc}
\hline CWPP subscales & FEIT & ToM $^{\mathrm{a}}$ & JTC $^{\mathrm{a}}$ & PAS $^{\mathrm{a}}$ & Neurocognitive \\
\hline Task orientation & $0.60^{* * *}\left(0.59^{* *}\right)$ & $0.12(0.03)$ & $0.07(0.13)$ & $-0.22(-0.18)$ & $0.37^{*}(0.36)$ \\
Social skills & $0.58^{* *}\left(0.55^{*}\right)$ & $0.28(0.12)$ & $-0.13(0.04)$ & $-0.31(-0.12)$ & $0.46^{*}\left(0.40^{*}\right)$ \\
Self-control & $0.42^{*}\left(0.39^{*}\right)$ & $0.09(-0.04)$ & $-0.03(0.00)$ & $-0.04(0.02)$ & $0.34(0.28)$ \\
Attitude & $0.36^{*}(0.35)$ & $0.16(0.13)$ & $0.08(0.16)$ & $-0.05(-0.04)$ & $0.44^{*}\left(0.43^{*}\right)$ \\
Personal presentation & $0.51^{* *}\left(0.45^{*}\right)$ & $-0.03(-0.15)$ & $0.17(0.16)$ & $0.04(0.02)$ & $0.25(0.14)$ \\
\hline
\end{tabular}

Note. The partial correlations for age is shown in brackets. FEIT $=$ Facial Expression Identification Test, ToM $=$ Theory of Mind, JTC $=$ Jump

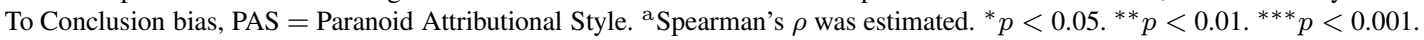

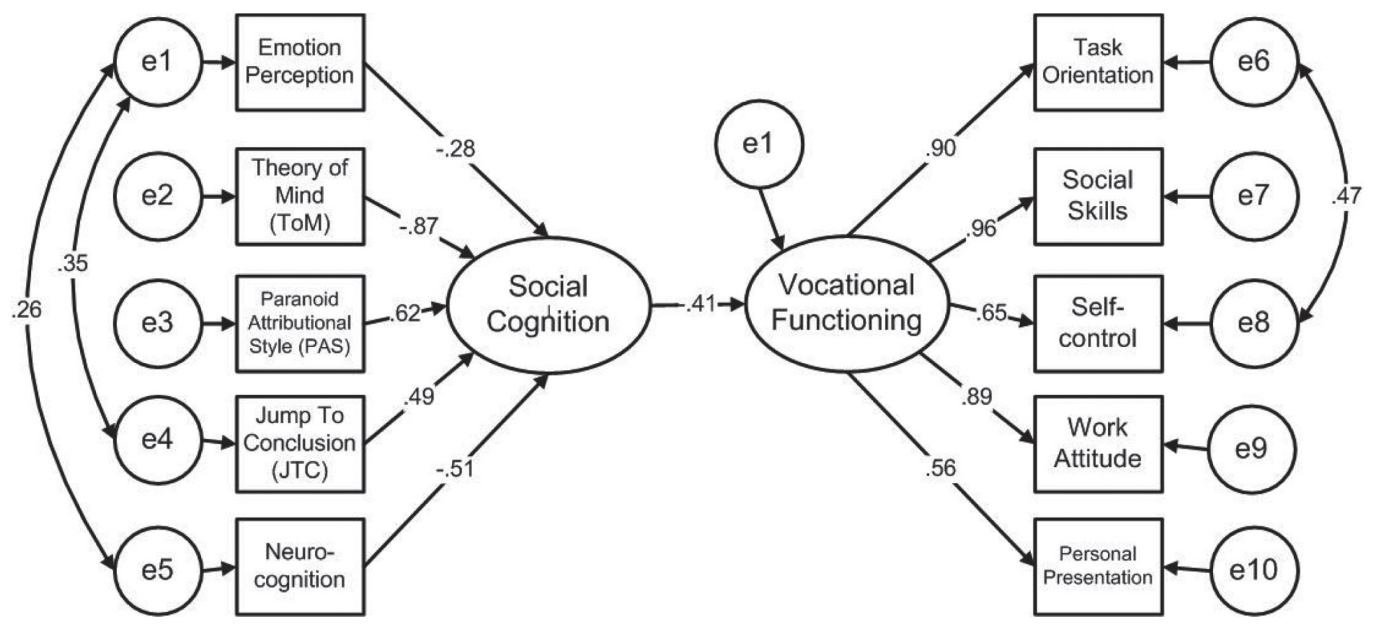

Fig. 1. Structural equation model on relationship between social cognition and vocational functioning.

to social-cognitive abilities are high, which also implies that both ToM and PAS play an important part in the mechanism of impact of social-cognitive abilities on vocational functioning. This is consistent with a previous meta-analysis which highlighted that ToM had the strongest relationship with global measures of community functioning or specific measures of activities of daily living, social skills, vocational functioning, and quality of life [16]. The adverse impact of PAS on vocational functioning is a relatively new finding.

Both these results and the findings of previous studies have consistently demonstrated a relatively strong relationship between ToM and vocational functioning in persons with schizophrenia, so one may question the need to assess other aspects of social cognition. We included other domains of social cognition in this study as we had hypothesized that ToM would not determine all aspects of work performance. For instance, among all the aspects of social cognition, EP is the only ability that relates to social behaviors [16]. The results have also revealed the importance of PAS, meaning that individuals with a hostile attributional style are more likely to experience difficulties in social relationships at work. The results of this study therefore pro- vide an opportunity to compare the effects of ToM with EP and attributional biases, and support that both ToM and paranoid attributions have an impact on work performance.

\subsection{Contributions and limitations}

This study makes several important contributions to our understanding of social-cognitive abilities in persons with schizophrenia. Firstly, it addresses the link between these abilities and work performance. The results demonstrate that social-cognitive ability, especially ToM, is the most important predictor of work performance. They also show that attributional errors have a negative impact. Secondly, this study has addressed some of the measurement issues found in previous work. Instead of using self-report measures and interviews, it has employed an observational assessment instrument to evaluate work performance in a simulated setting. This is more likely to provide objective and reliable estimates of vocational functioning than the measures used in previous studies. When compared with previous studies, a more comprehen- 
sive evaluation of social cognition was conducted in this study, including ToM, attributional biases, and EP.

However, there are also several limitations to our study. Firstly, there are few assessment tools available for the comprehensive evaluation of social-cognitive abilities. The newly-available C-SCSQ is one of the few such assessments that has a Chinese version. It is a brief assessment (taking 30 to 40 minutes) and the ToM, JTC, and PAS subscales tend to be highly correlated as they are obtained from the examinee's answers to different questions anchored on the same case vignettes (ambiguous social situations). Secondly, some researchers regard ToM as a comprehensive construct encompassing various levels of social-cognitive abilities from understanding indirect speech and detection of lies to intention referencing. While it is commonly a focus of studies of social-cognitive abilities, there are still ongoing discussions on how it should be assessed either in persons with schizophrenia or in healthy populations. The measurement tool used in this study mainly assesses ToM using the intentionreferencing approach. Thirdly, it should be noted that the neurocognitive measure in C-SCSQ is not a standard neurocognitive test and requires some level of social knowledge and the use of inference to answer some of the questions related to the social situations in the vignettes. It is designed to assess memory among all the neurocognitive measures. Lastly, this study is cross-sectional in design and thus the structural equations model may highlight associations rather causations. A prospective or cohort study could be used to trace the vocational outcomes for individual clients and examine how social-cognitive abilities and biases may play a part in determining such outcomes.

\section{Conclusion}

The results of this study have showed that social cognition has a significant impact on vocational functioning. Of the four social-cognitive domains, ToM contributes to a significant proportion of the variance in such performance. The results support the proposition that there is a close relationship between social cognition and vocational functioning, and underscore the importance of training in such cognitive attributes as part of vocational rehabilitation. Future studies should adopt a prospective design to further confirm the contribution of social-cognitive abilities to vocational outcomes.

\section{References}

[1] Dickerson D, Bellack AS, Gold JM. Ratings of social functioning in outpatients with schizophrenia: Patient self-report versus caregiver assessment. Eval Program Plann. 1997; 20: 415-420.

[2] Jaeger J, Berns SM, Czobor P. The Multidimensional Scale of Independent Functioning: A new instrument for measuring functional disability in psychiatric populations. Schizophrenia Bull. 2003; 29: 153-168.

[3] Wallace CJ. Functional assessment in rehabilitation. Schizophrenia Bull. 1986; 12(4): 604-630.

[4] Strong S. Meaningful work in supportive environments: Experiences with the recovery process. Am J Occup Ther. 1998; 52: 31-38.

[5] Laird M, Krown S. Evaluation of a transitional employment program. Psychosoc Rehabil J. 1991; 15: 3-8.

[6] Bell MD, Milstein RM, Licker PH. Pay as an incentive in work participation by patients with severe mental illness. Hosp Community Psych. 1993; 44: 684-686.

[7] Bell MD, Lysaker PH, Milstein RM. Clinical benefits of paid work activity in schizophrenia. Schizophrenia Bull. 1996; 22(1): 51-67.

[8] Equal Opportunities Council of Hong Kong [homepage on the Internet]. Hong Kong: A baseline survey on employment situation of persons with a disability in Hong Kong [updated 1997; cited 2013 Feb 15] Available from: http:// www.eoc.org.hk/eoc/GraphicsFolder/default.aspx.

[9] Westwood R, Lok P. The meaning of work in a Chinese context a comparative study. Int J Cross Cult Man. 2003; 3: 139165.

[10] Bell MD, Bryson G. Work Rehabilitation in Schizophrenia: Does Cognitive Impairment Limit Improvement. Schizophrenia Bull. 2001; 27: 269-279.

[11] Green MF. What are the functional consequences of neurocognitive deficits in schizophrenia? Am J Psychiat. 1996; 153: 321-330.

[12] Velligan DJ, Bow-thomas CC, Mahurin RK, Miller AL, Halgunseth LD. Do specific neurocognitive deficits predict specific domains of community function in schizophrenia? J Nerv Ment Dis. 2000; 188: 518-524.

[13] Couture SM, Penn DL, Roberts DL. The functional significance of social cognition in schizophrenia: a review. Schizophrenia Bull. 2006; 32: 44-63.

[14] Schmidt SJ, Mueller DR, Roder V. Social cognition as a mediator variable between neurocognition and functional outcome in schizophrenia: Empirical review and new results by structural equation modeling. Schizophrenia Bull. 2011; 37: 41-54.

[15] Brekke J, Kay DD, Lee KS, Green MF. Biosocial pathways to functional outcome in schizophrenia. Schizophr Res. 2005; 80: 213-225.

[16] Fett AK, Viechtbauer W, Dominguez M, Penn DL, Os JV, Krabbendam $L$. The relationship between neurocognition and social cognition with functional outcomes in schizophrenia: A meta-analysis. Neurosci Biobehav R. 2011; 35: 573-588.

[17] Pijenborg GHM, Withaar FK, Evans JJ, Bosh VD, Timmerman ME, Brouwer WH. The predictive value of measures of social cognition for community functioning in schizophrenia: Implications for neuropsychological assessment. J Int Neuropsych Soc. 2009; 15: 239-247.

[18] Fiske ST, Taylor SE. Social cognition 2nd ed. New York, NY: McGraw-Hill, 1991; p. 14-19.

[19] Penn DL, Van der DA, Spaulding WD, Garbin C, Linszen $\mathrm{D}$, Dingemans $\mathrm{P}$. Information processing and social cognitive 
problem solving in schizophrenia: Assessment of interrelationships and changes over time. J Nerv Ment Dis. 1993; 181: 13-20.

[20] Adolphs R. Investigating the cognitive neuroscience of social behavior. Neuropsychologia. 2003; 41: 119-126.

[21] Pinkham AE, Penn DL, Perkins DO, Lieberman J. Implications for the neural basis of social cognition for the study of schizophrenia. Am J Psychiat. 2003; 160: 815-824.

[22] Bryson G, Bell MD, Lysaker PH. Affect recognition in schizophrenia: a function of global impairment or a specific cognitive deficit. Psychiat Res. 1997; 71: 105-113.

[23] Mehta UM, Thirthalli J, Subbakrishna DK, Gangadhar BN Eack SM, Keshavan MS. Social and neuro-cognition as distinct cognitive factors in schizophrenia: a systematic review. Schizophr Res. c2013 [updated 2013 May 31; cited 2013 Jun 13] Available from: http://dx.doi.org/10.1016/j.schres. 2013.05.009.

[24] Green MF, Penn DL, Bentall R, Carpenter, WT, Gaebel W, Gur RC, Kring AM, Park S, Silverstein SM, Heinssen R. Social cognition in schizophrenia: An NIMH Workshop on definitions, assessment, and research opportunities. Schizophrenia Bull. 2008; 24: 1211-1220.

[25] Roberts DL, Penn DL. Social cognition and interaction training (SCIT) for outpatients with schizophrenia: A preliminary study. Psychiatry Res. 2009; 166: 141-147.

[26] Kohler CG, Walker JB, Martin EA, Healey KM, Moberg PJ. Facial emotion perception in schizophrenia: A meta-analytic review. Schizophrenia Bull. 2010; 36: 1009-1019.

[27] Kohler CG, Turner TH, Bilker WB, Brensinger CM, Siegel SJ, Kanes SJ, et al. Facial emotion recognition in schizophrenia: Intensity effects and error pattern. Am J Psychiat. 2003; 160: 1768-1774

[28] Premack D, Woodruff G. Does the chimpanzee have a theory of mind? Behav Brain Sci. 1978; 1: 515-526.

[29] Corcoran R, Mercer G, Frith CD. Schizophrenia, symptomatology and social inference: Investigating 'theory of mind' in people with schizophrenia. Schizophr Res. 1995; 17: 5-13.

[30] Frith CD, Corcoran R. Exploring 'theory of mind' in people with schizophrenia. Psychol Med. 1996; 26: 521-530.

[31] Mazza M, De Risio A, Surian L, Roncone R, Casacchia M. Selective impairments of theory of mind in people with schizophrenia. Schizophr Res. 2001; 47: 299-308.

[32] Sprong M, Schothorst P, Vos E, Hox J, Engeland HV. Theory of mind in schizophrenia: Meta-analysis. Brit J Psychiat. 2007; 191: 5-13.

[33] Combs DR, Penn DL, Michael CO, Basso MR, Wiedeman $\mathrm{R}$, Siebenmorgan M, et al. Perceptions of hostility by persons with and without persecutory delusions. Cogn Neuropsychiatry. $2009 ; 14: 30-52$

[34] Freeman D. Suspicious minds: The psychology of persecutory delusions. Clin Psychol Rev. 2007; 27: 425-457.

[35] McKay R, Langdon R, Coltheart M. Need for closure, jumping to conclusions, and decisiveness in delusion-prone individuals. J Nerv Ment Dis. 2006; 194: 422-426.

[36] Mancuso F, Horan WP, Kern RS, Green MF. Social cognition in psychosis: Multidimensional structure, clinical co- rrelates, and relationship with functional outcome. Schizophr Res. 2011; 125: 143-151.

37] Vauth R, Rusch N. Wirtz M, Corrigan PW. Does social cognition influence the relation between neurocognitive deficits and vocational functioning in schizophrenia? Psychiat Res. 2004; 128: $155-165$.

[38] Bell M, Tsang HWH, Greig TC, Bryson GJ. Neurocognition, social cognition, perceived social discomfort, and vocational outcomes in schizophrenia. Schizophrenia Bull. 2009; 35: 738-747.

[39] Couture SM, Granholm EL, Fish SC. A path model investigation of neurocognition, theory of mind, social competence, negative symptoms and real-world functioning in schizophrenia. Schizophr Res. 2011; 125: 152-160.

[40] Gard DE, Fisher M, Garrett C, Genesky A, Vinogradov S. Motivation and its relationship to neurocognition, social cognition, and functional outcome in schizophrenia. Schizophr Res. 2009; 115: 74-81.

[41] McKibbin CL, Brekke JS, Sires D, Jeste DV, Patterson TL. Direct assessment of functional abilities: relevance to persons with schizophrenia. Schizophr Res. 2004; 72: 53-67.

[42] Angermeyer MC, Goldstein JM, Kuehn L. Gender differences in schizophrenia: rehospitalization and community survival. Psychol Med. 1989; 19: 365-382.

[43] Goldstein JM, Seidman LJ, Goodman JM, Koren D, Lee H, Weintraub S, et al. Are there sex differences in neuropsychological functions among patients with schizophrenia? Am J Psychiat. 1998; 155: 1358-1364.

[44] Law CKM, Siu AMH, Lee JLY, Lee WK. Prediction of work rehabilitation placements using the Chinese Work Personality Profile. Psychiatr Rehabil J. 2006; 30: 120-128.

[45] Kerr SL, Neale JM. Emotion perception in schizophrenia: Specific deficit or further evidence of generalized poor performance? J Abnorm Psychol. 1993; 102: 312-318.

[46] Matsumoto D, Ekman P. Japanese and Caucasian facial expression of emotion (JACFEE) and neutral faces (JACNeuF). [Assessment Manual]. San Francisco, CA: University of California; 1988.

[47] Yip JTH, Lee TMC. Cultural differences in facial emotion recognition. Brain Cognition. 2003; 51: 169-170.

[48] Mueser KT, Doonan R, Penn DL, Blanchard JJ, Bellack AS, Nishith P, et al. Emotion recognition and social competence in chronic schizophrenia. J Abnorm Psychol. 1996; 105: 271275.

[49] Addington J, Saeedi H, Addington D. Facial affect recognition: A mediator between cognitive and social functioning in psychosis. Schizophr Res. 2006; 85: 142-150.

[50] Addington J, Penn D, Woods SW, Addington D, Perkin DO. Facial affect recognition in individuals at clinical high risk for psychosis. Brit J Psychiat. 2008; 192: 67-68.

[51] Bora M, Yucel M, Panetils C. Theory of mind impairment in schizophrenia: Meta-analysis. Schizophr Res. 2009; 109: 1-9.

[52] Sergi MJ, Rassovsky Y, Widmark C, Reist C, Erhart S, Braff D, et al. Social cognition in schizophrenia: Relationships with neurocognition and negative symptoms. Schizophr Res. 2007; 90: $316-324$ 